\title{
Factores biopsicológigos y socioculturales como predictores de la conducta promotora de salud en los estudiantes de Enfermería de la Universidad Adventista de Chile
}

\author{
Biopsicológigos and sociocultural factors as predictors of health promoting behavior of nursing \\ students of the Adventist University of Chile
}

Daysi Aranda Sánchez¹, Mónica Cruz Pedreros²

\begin{abstract}
RESUMEN
Objetivo: Determinar si los factores biológicos, psicológicos y socioculturales de los estudiantes de Enfermería de la Universidad Adventista de Chile son predictores de las conductas promotoras de salud (CPS). Metodología: Estudio cuantitativo, descriptivo-correlacional, de corte transversal. La población: todos los estudiantes del primer al quinto año de la carrera de Enfermería. La muestra fue de 137 alumnos, seleccionada con un muestreo probabilístico aleatorio, estratificado. El instrumento estuvo conformado por un cuestionario semiestructurado, escala de estilo de vida promotor de salud (EVPS), escala de percepción de autoeficacia y escala de autoestima. Los datos fueron procesados con SPSS 19.0. Resultados: Los factores biosocioculturales no son predictores de las conductas promotoras de salud en los estudiantes de Enfermería, pues fueron evaluados a un nivel de confianza de $95 \%$ sin embargo, el factor psicológico autoestima presentó una asociación con la conducta promotora de salud, como lo indica el valor $\mathrm{X}^{2}=23.56, \mathrm{p}=0.00$. y el factor percepción de autoeficacia, una relación directa, significativa con la conducta promotora de salud, $r=0.30, p=0.00$. Conclusión: La autoestima y la autoeficacia percibida son predictores de la conducta promotora de salud en los estudiantes de Enfermería de la Universidad Adventista de Chile.
\end{abstract}

Palabras Clave: Conducta promotora de salud, factores predictores, estudiantes de enfermería.

\begin{abstract}
Objective: To determine the biological, psychological and sociocultural factors of nursing students of the Adventist University of Chile are predictors of health promoting behaviors (CPS). Methodology: descriptivecorrelational quantitative cross-sectional study. Population: all first through fifth year of the undergraduate course. The sample was 137 students, selected by a random probability sampling, stratified. The instrument consisted of a semistructured questionnaire, level of lifestyle health promoter (EVPS) scale of perceived self-efficacy and self-esteem scale. Data were processed with SPSS 19.0. Results: biosocioculturales factors are not predictors of health promoter behaviors in nursing students, as were evaluated at a confidence level of $95 \%$ however, the self psychological factor provided an association with promoting health behavior, as indicates the value $\mathrm{X} 2=23.56, \mathrm{p}$ $=0.00$. and self-efficacy factor, has a direct and positive relationship with health promoting behavior, $r=0.30, p$ $=0.00$. Conclusion: Self-esteem and self-efficacy are predictors of health promoting behavior in nursing students of the Adventist University of Chile.
\end{abstract}

Keywords: Health-promoting behavior, predictors, nursing students.

\footnotetext{
${ }^{I}$ Docente en la Unidad de Posgrado de Ciencias de la Salud, Universidad Peruana Unión, Lima

${ }^{2}$ Docente de la Escuela de Enfermería de la Universidad de Concepción de Chile

Correspondencia: daysi_aranda@hotmail.com
} 


\section{INTRODUCCIÓN}

La evolución del sistema organizacional mundial presentado por la urbanización, la industrialización y la tendencia a la globalización, ha condicionado que, en los países en vías de desarrollo, se produzcan cambios en los estilos de vida de las personas, tornándolos poco saludables; potenciando así el desarrollo de las enfermedades crónicas no transmisibles. En respuesta a lo anterior, en el 2005 se llevó a cabo la Sexta Conferencia Mundial de Promoción de la Salud realizada en Bangkok, obteniendo como resultado la "Carta de Bangkok para el Fomento de la Salud en un Mundo Globalizado", donde se enfatiza la necesidad de capacitar a las personas, ejerciendo un mayor control sobre los factores influyentes que predicen la salud (OPS, 2005 \& OMS, 2006). En Chile, la Encuesta Nacional de Salud del 2003 informa la prevalencia de riesgo cardiovascular alto y muy alto en el nivel universitario con un 49,6\%; la prevalencia de tabaquismo y sedentarismo con un $85 \%$ y $46,7 \%$ respectivamente. Aunque la población universitaria representa un capital humano importante para la sociedad, las propias universidades y las políticas de salud del MINSAL carecen de estrategias concretas que favorezcan la promoción de la salud en esta población en riesgo (Aspillaga, 2003).

El estilo de vida del universitario implica cambios en la dimensión biopsicosociocultural que pueden repercutir negativamente en la salud. El estudiante se enfrenta a una etapa en que el tiempo es limitado para consumir sus alimentos de manera equilibrada, la demanda del consumo de comida rápida es cada vez más alta y la actividad física escasa; el aumento de consumo de alcohol y tabaco se hacen evidentes por la necesidad que tienen los jóvenes de vivir su libertad fuera de la familia y la influencia de los compañeros sobre su individualidad (MINSAL, 1997). Los estudiantes universitarios de la carrera de Enfermería no están ajenos a estas conductas de riesgo, pese a su formación. Por ello, es necesario identificar factores que pueden predecir la conducta de salud para fomentar estrategias en su formación académica que los conduzcan a realizar esfuerzos preventivopromocionales en el cuidado de la salud. En esa perspectiva, se plantea la siguiente pregunta: ¿Son los factores biosociológicos y socioculturales predictores de las conductas promotoras de salud en los estudiantes Enfermería de la Universidad Adventista de Chile?

Este estudio se sustenta en el Modelo de Promoción de Salud (MPS) de Nola Pender (Figura 1) quien, al referirse a los estilos de vida, incluye el concepto de Conductas Promotoras de Salud. El MPS es un esquema que integra constructos de la teoría de expectativasvalores y de la teoría cognitivo-social, otorgando una solución de Enfermería a la política sanitaria y la reforma del cuidado de salud, identificando la estructura multidimensional que predicen las conductas de las personas respecto a la salud, ofreciendo un medio para comprender cómo pueden verse motivados a alcanzar la salud y bienestar en interacción con su ambiente.

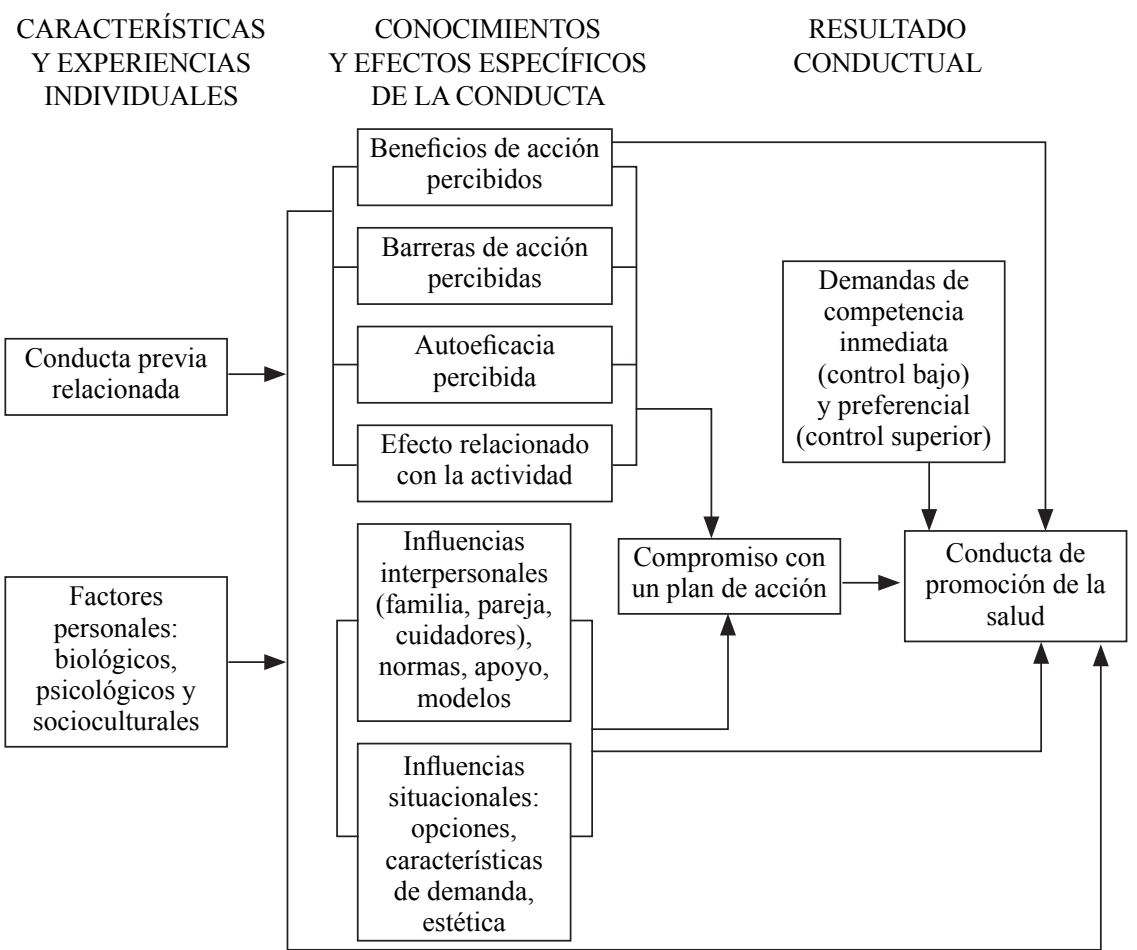

Figura 1

Modelo de Promoción de La Salud, 2006. 


\section{MATERIAL Y MÉTODOS}

Investigación de abordaje cuantitativo, descriptivo - correlacional de corte transversal. La muestra estuvo conformada por 137 alumnos del primer al quinto año de la carrera de Enfermería de la Universidad Adventista de Chile, seleccionada con un muestreo de tipo probabilístico aleatorio, estratificado, siendo el año académico criterio de estratificación. Para la recolección de datos se utilizó: Un cuestionario semiestructurado realizado por la autora para medir los factores socioculturales, la Escala de Estilo de vida Promotor de Salud II (EVPS) de (Walker, Kerr, Sechrist y Pender, citado por Cid, Merino y Stiepovich 2006) que representan conductas o acciones en cada una de las subescalas: crecimiento espiritual, manejo del estrés, actividad física, apoyo interpersonal, nutrición y responsabilidad en salud; con respuesta a escala Likert. La escala de Percepción de Autoeficacia de (Schwarzer y Babler, 1996; citado por Cid, Merino y Stiepovich 2006) que mide la percepción que tiene la persona respecto a sus capacidades. La escala de Autoestima de Morris Rosenberg (1965), esta escala mide la valoración que hace el individuo acerca de su propia persona y la escala de Percepción del Estado de Salud: elaborada por (Reker y Wong en 1984; citado por Cid, Merino y Stiepovich 2006) permite que la persona encuestada autovalore su estado de salud, y se subdivide en dos subescalas: salud física con 8 ítems y los otros 8 ítems salud psicológica.

Para medir la confiabilidad de las escalas, se utilizó el coeficiente Alpha de Cronbach, en una prueba piloto a un grupo de 38 alumnos pertenecientes del primer al quinto año de la carrera de Enfermería, de la Universidad de Concepción, que cumplen con características similares establecidas en la población en estudio. Los resultados obtenidos fueron: para la Escala Estilo de Vida Promotor de Salud II (EVPS) el Alpha de Cronbach fue de 0,82 para las escalas: Autoestima, Percepción de Autoeficacia y Percepción del estado de Salud, se obtuvieron de: 0.87. 0.86 y 0.49 respectivamente. En el procesamiento y análisis de los datos se utilizó el programa estadístico SPSS versión 19, considerándose: El análisis descriptivo de cada una de las variables de estudio y las técnicas de estadística inferencial, para identificar los factores predictores de las conductas promotoras de salud.

\section{RESULTADOS}

\section{Tabla 1}

Características descriptivas de la variable dependiente: Conducta Promotora de Salud en los estudiantes de Enfermería de la Universidad Adventista de Chile.

\begin{tabular}{cc}
\hline Características Descriptivas & Estadísticos \\
\hline N & 132 \\
Media & 139.8 \\
Mediana & 140.5 \\
Moda & 141 \\
Desviación estándar & 15.1 \\
Rango & 96 \\
Percentiles 30/80 & $132 / 153$ \\
\hline
\end{tabular}

Tabla 2

Categorización de la variable Conducta Promotora de Salud (CPS)

\begin{tabular}{cccc}
\hline Conducta Promotora de Salud (CPS) & Frecuencia & Porcentaje válido & Porcentaje acumulado \\
\hline $\begin{array}{c}\text { Baja CPS } \\
\text { (<132 puntos })\end{array}$ & 42 & 31.8 & 31.8 \\
$\begin{array}{c}\text { CPS Media } \\
(132-153 \text { puntos })\end{array}$ & 63 & 47.7 & 79.5 \\
Alta CPS (> 153 puntos $)$ & 27 & 20.5 & 100.0 \\
Total & $132^{*}$ & 100 & \\
\hline & *Valores perdidos 6.
\end{tabular}


La distribución descriptiva de los puntajes obtenidos de la variable dependiente conducta promotora de salud (CPS) mostrada en la tabla $\mathrm{N}^{\circ} 1$ indica que: el puntaje promedio de la CPS fue de 139.8. El puntaje que más se repitió fue de 141 puntos. El puntaje mínimo fue de 92 y el máximo de 188, mostrando amplia variabilidad de la muestra. La categorización de los puntajes, según cálculos de percentiles para la variable CPS (tabla N. ${ }^{\circ}$ ), califica a los puntajes bajo el percentil 30 como baja CPS; sobre el percentil 80 como alta CPS, y los puntajes que fluctúan entre los percentiles 30 y 80 como CPS de categoría media. Observándose, entonces, que un $47.7 \%$ presentaron CPS de categoría media.

\section{Análisis inferencial bivariada entre variable dependiente y variables independientes.}

Tabla 3

Conducta Promotora de Salud según edad de los estudiantes de Enfermería de la Universidad Adventista de Chile.

\begin{tabular}{cccccc}
\hline & \multicolumn{3}{c}{ Conducta Promotora de Salud } & \multicolumn{2}{c}{ ANOVA } \\
\hline Edad & $\mathrm{n}$ & Media & Desv. Tip. & $\mathrm{F}$ & Sig. (bil) \\
& & & & 0.22 & 0.80 \\
(No sig.) \\
<20 años & 38 & 139.76 & 14.20 & & \\
$20-24$ años & 53 & 140.83 & 14.98 & & \\
$>24$ años & 41 & 138.73 & 16.38 & \\
Total & 132 & 139.87 & 15.1 & \\
\hline
\end{tabular}

La tabla 3 muestra que los estudiantes en edades de 20 a 24 años obtuvieron el más alto promedio de conducta promotora de salud con 140.9 puntos; los estudiantes con menos de 20 años obtuvieron un promedio de 139.7 puntos; así, y los estudiantes con más de 24 años obtuvieron el menor promedio de conducta promotora de salud con 138.7 puntos. Estas diferencias en los puntajes promedios no son estadísticamente significativas como lo indica el valor $p>0.05$. Se infiere por lo tanto, que la edad no es un factor predictivo en la conducta promotora de salud en los estudiantes.

Tabla 4

Conducta Promotora de Salud según sexo.

\begin{tabular}{|c|c|c|c|c|c|}
\hline \multirow[b]{2}{*}{ Sexo } & \multicolumn{3}{|c|}{ Conducta Promotora de Salud } & \multicolumn{2}{|c|}{ Prueba T } \\
\hline & $\mathrm{N}$ & Media & Desv. Tip. & $\mathrm{T}$ & Sig. (bil) \\
\hline & & & & 1.504 & $\begin{array}{c}0.135 \\
\text { (No sign.) }\end{array}$ \\
\hline Femenino & 104 & 140.89 & 15.32 & & \\
\hline Masculino & 28 & 136.07 & 14.00 & & \\
\hline Total & 132 & & & & \\
\hline
\end{tabular}

La tabla 4 se observa que los estudiantes del sexo femenino $(n=104)$ son las que obtuvieron el puntaje promedio más alto de la conducta promotora de salud con 140.8 puntos, respecto a los estudiantes del sexo masculino $(\mathrm{n}=28)$ que obtuvieron un puntaje promedio de 136.07 . Con un $95 \%$ de confianza, el cálculo de la prueba t resultó un valor de $\mathrm{p}>0.05$, indicando que el sexo de los estudiantes de Enfermería no es un factor predictor de la conducta promotora de salud. 
Factores biopsicológigos y socioculturales como predictores de la conducta promotora de salud en los estudiantes de Enfermería de la Universidad Adventista de Chile

Tabla 5

Conducta Promotora de Salud según año académico

\begin{tabular}{llllll}
\hline & \multicolumn{2}{l}{ Conducta Promotora de Salud } & \multicolumn{3}{l}{ ANOVA } \\
\hline Año académico & $\mathrm{N}$ & Media & Desv. Tip. & $\mathrm{F}$ & \multicolumn{1}{l}{ Sig. (bil) } \\
& & & & 1.257 & 0.29 (No sign.) \\
Primero & 39 & 138.61 & 15.98 & & \\
Segundo & 30 & 138.66 & 13.12 & & \\
Tercero & 34 & 138.88 & 15.43 & & \\
Cuarto & 20 & 146.80 & 15.81 & & \\
Quinto & 9 & 137.66 & 13.77 & & \\
Total & 132 & 139.87 & 15.12 & & \\
\hline
\end{tabular}

La tabla 5 muestra que el promedio más alto de CPS lo obtuvieron los estudiantes del cuarto año con 146.80 puntos. Los promedios del primero al quinto no son estadísticamente significativos, como lo indica $\mathrm{p}>0.05$. De lo anterior se infiere que el año académico no predice en la conducta promotora de salud.

Tabla 6

Conducta Promotora de Salud según procedencia

\begin{tabular}{llllll}
\hline & \multicolumn{2}{l}{ Conducta Promotora de Salud } & \multicolumn{2}{l}{ Prueba T } \\
\hline Procedencia & $\mathrm{n}$ & Media & Desv. Tip. & $\mathrm{t}$ & Sig. (bil) \\
& & & & 0.078 & 0.93 (No sign.) \\
Rural & 18 & 139.61 & 14.08 & & \\
Urbano & 114 & 139.91 & 15.34 & & \\
Total & 132 & & & & \\
\hline
\end{tabular}

La tabla 6 muestra que los estudiantes de Enfermería, que proceden de la zona rural, obtuvieron un promedio de 139.61 y los que proceden de la zona urbana obtuvieron un promedio de 139.91 puntos, calificados como CPS de categoría media.
Las diferencias en los puntajes promedios en la escala CPS, según procedencia, no son estadísticamente significativas como lo indica el valor de $p>0.05$ en la prueba t. entonces se infiere que la procedencia de los estudiantes no es un factor predictor de la CPS.

Tabla 7

Conducta Promotora de Salud según convivencia.

\begin{tabular}{cccccc}
\hline & \multicolumn{3}{c}{ Conducta Promotora de Salud } & \multicolumn{2}{c}{ ANOVA } \\
\hline Convivencia & $\mathrm{n}$ & Media & Desv. Tip. & $\mathrm{F}$ & $\begin{array}{c}\text { Sig. } \\
\text { (No sign.) } \\
\text { Solo }\end{array}$ \\
& 7 & 144.57 & 27.93 & & \\
$\begin{array}{c}\text { Amigos y } \\
\text { compañeros }\end{array}$ & 25 & 135.32 & 15.99 & & \\
Padres o familiares & 100 & 140.68 & 13.64 & & \\
Total & 132 & 139.87 & 15.12 & & \\
\hline
\end{tabular}


En la tabla 7 se observa que los estudiantes, que viven solos, obtuvieron el más alto promedio de la CPS alcanzando 144.57 puntos y los estudiantes que indicaron vivir con amigos y compañeros alcanzaron el más bajo promedio de 135.32 puntos. Estas diferencias de puntajes promedios en la conducta promotora de salud, según convivencia, no son estadísticamente significativas: $p>0.05$. Por lo que se infiere que la variable convivencia no predice la conducta promotora de salud.

\section{Tabla 8}

Conducta Promotora de Salud según religión.

\begin{tabular}{cccccc}
\hline & \multicolumn{3}{c}{ Conducta Promotora de Salud } & \multicolumn{2}{c}{ Prueba T } \\
\hline Religión & $n$ & Media & Desv. Tip. & $t$ & Sig. (bil) \\
& & & & 1.74 & 0.08 \\
(No sign.) & & \\
Con religión & 124 & 140.45 & 14.87 & \\
Sin religión & 8 & 130.87 & 17.19 & \\
Total & 132 & & & & \\
\hline
\end{tabular}

La tabla 8 muestra que los estudiantes, que indicaron profesar alguna religión, obtuvieron un promedio de 140.45 puntos, categorizada como CPS media respecto a los estudiantes que indicaron no profesar alguna religión los que obtuvieron un promedio de 130.87 puntos, categorizada como
CPS baja. Es decir los estudiantes que indicaron profesar alguna religión tienen mejores CPS que los estudiantes que no indicaron profesar alguna religión, sin embargo, esta variable no predice en la conducta promotora de salud de los estudiantes en estudio, como lo indica $\mathrm{p}>0.05$.

Tabla 9

Conducta Promotora de Salud según estado de pareja.

\begin{tabular}{|c|c|c|c|c|c|}
\hline \multirow{3}{*}{ Estado de pareja } & \multicolumn{3}{|c|}{ Conducta Promotora de Salud } & \multicolumn{2}{|c|}{ Prueba T } \\
\hline & $\mathrm{n}$ & Media & Desv. Tip. & $\mathrm{t}$ & Sig. (bil) \\
\hline & & & & 1.74 & 0.93 (No sign.) \\
\hline Con pareja & 64 & 141.95 & 15.94 & & \\
\hline Sin pareja & 68 & 137.91 & 14.15 & & \\
\hline Total & 132 & & & & \\
\hline
\end{tabular}

En la tabla 9 se observa que, los estudiantes que indicaron tener pareja, presentaron un promedio de 141.95 puntos, respecto al promedio de estudiantes que no tienen pareja con 137.91 puntos. El estado de pareja no predice la conducta promotora de salud como lo indica $\mathrm{p}>0.05$.

Tabla 10

Conducta Promotora de Salud según autoestima de los estudiantes de enfermería

\begin{tabular}{|c|c|c|c|c|c|}
\hline \multirow{2}{*}{ Nivel de Autoestima } & & \multicolumn{3}{|c|}{ Conducta Promotora de Salud } & \multirow{2}{*}{ Tota } \\
\hline & & CPS baja & CPS media & CPS alta & \\
\hline \multirow{3}{*}{ Autoestima Baja } & $\mathrm{n}$ & 26 & 16 & 6 & 48 \\
\hline & $\%$ & 54.2 & 33.3 & 12.5 & 100 \\
\hline & $\mathrm{n}$ & 11 & 29 & 6 & 46 \\
\hline \multirow[t]{2}{*}{ Autoestima Media } & $\%$ & 23.9 & 63.3 & 13.0 & 100 \\
\hline & $\mathrm{n}$ & 5 & 18 & 13 & 36 \\
\hline \multirow[t]{2}{*}{ Autoestima Alta } & $\%$ & 13.9 & 50.0 & 36.1 & 100 \\
\hline & $\mathrm{n}$ & 42 & 63 & 25 & 130 \\
\hline Total & $\%$ & 32.3 & 48.5 & 19.2 & 100 \\
\hline
\end{tabular}


La tabla 10 y 11 indican el nivel de CPS según los niveles de autoestima en los estudiantes de enfermería. Del $100 \%$ de estudiantes con autoestima baja un $54.2 \%$ presenta CPS baja, del total de participantes con autoestima media un $63.3 \%$ presenta CPS en el nivel medio y el 50\% de estudiantes con autoestima alta presenta un CPS de nivel medio. Además la prueba Chi cuadrado indica una asociación significativa con $\mathrm{X}^{2}=23.56, \mathrm{P}=0.00$ con un nivel de confianza del $95 \%$.

Tabla 11

Asociación entre la Conducta Promotora de Salud y el nivel de autoestima.

\begin{tabular}{ccc}
\hline Prueba & Valor & Sig. \\
\hline Chi cuadrado $\left(\mathrm{x}^{2}\right)$ & 23.56 & 0.00 \\
\hline Coeficiente & 0.39 & \\
\hline
\end{tabular}

Tabla 12

Conducta Promotora de Salud según percepción de autoeficacia

\begin{tabular}{|c|c|c|c|}
\hline & Media & Desv. Típica & N \\
\hline CPS & 139.96 & 15.22 & 130 \\
\hline Percepción de autoeficacia & 32.97 & 4.59 & 130 \\
\hline$r$ de Pearson & 0.30 & \multicolumn{2}{|c|}{$P=0.00$} \\
\hline $\mathrm{R}^{2}$ & 0.09 & \multicolumn{2}{|c|}{$P=0.00$} \\
\hline B no estándar & 0.99 & \multicolumn{2}{|c|}{$P=0.00$} \\
\hline $\mathrm{t}$ & 3.5 & & \\
\hline
\end{tabular}

En la tabla 12 se observa un puntaje promedio de 139.96 de la CPS y un promedio de 32.97 percepción de autoeficacia. El análisis estadístico mostró una asociación directa con $\mathrm{r}=0.30, \mathrm{P}=0.00$ lo que indica que a mayor percepción de autoeficacia mayor conducta promotora de salud presentada por los estudiantes de enfermería.

\section{DISCUSIÓN}

Los resultados en esta investigación muestran que el $25.5 \%$ de los estudiantes de Enfermería, del primer al quinto año estuvo orientado a niveles más altos de salud (CPS alta) respecto a los estudiantes que presentaron un nivel bajo de CPS que fue de $31.8 \%$, siendo esta última, población vulnerable a adquirir enfermedades crónicas no trasmisibles como: obesidad, diabetes mellitus II, hipertensión, dislipidemias, estrés y otras. No obstante, cerca del $50 \%$ de los estudiantes, presentó CPS de categoría media, significando que, con estrategias de acción efectivas y certeras en la promoción de conductas saludables, sería posible optimizar sus conductas promotoras de salud y mejorar su calidad de vida.

A nivel nacional no se han encontrado estudios específicos que midan CPS en estudiantes universitarios, sin embargo, estudios realizados en comunidades chilenas muestran resultados de conductas promotoras de salud en el nivel superior, así Cid (2008), encontró que las personas de escolaridad superior, del sector Barrio Norte, presentaron CPS similares a los de escolaridad baja. Otro estudio realizado en Chile por (Chávez, 2009; citado por Cid, Merino y Stiepovich 2006) en la Comuna de Coronel, encontró que las personas de nivel de escolaridad superior completa e incompleta, presentan mejores conductas promotoras de salud que los de nivel de escolaridad media y básica, concluyendo que el nivel de escolaridad influye en las conductas promotoras de salud. En cuanto a las variables biológicas: la edad no resultó variable predictora de la CPS en los estudiantes de Enfermería, sin embargo, el mayor puntaje en conducta promotora de salud lo obtuvieron los estudiantes de 20 a 24 años. Este hallazgo encuentra apoyo en el estudio de calidad y estilos de vida en 
estudiantes de Enfermería realizado por González (2004); donde reporta que los alumnos con edades entre 22,7 y 24,5 años, presentan conductas que se direccionan hacia estilos de vida sanos. Es importante resaltar que, a nivel nacional, la población joven de 20 a 24 años ocupa el tercer lugar en Chile, según el informe dado por el sistema nacional de estadísticas vitales, 2007.

El sexo de los estudiantes tampoco tuvo influencia significativa, sin embargo, las estudiantes presentaron mejores conductas promotoras de salud con respecto a los estudiantes varones, resultado que coincide con el estudio realizado por (Chávez, 2007; citado por Cid, Merino y Stiepovich 2006) quienes midieron las mismas conductas en una población, donde las mujeres presentaron mejores conductas promotoras de salud que los hombres. El tipo de convivencia de los estudiantes de Enfermería no es una variable predictora de la conducta promotora de salud, en este estudio el estudiante que vive solo, presenta mejores CPS que los estudiantes que viven con sus amigos o compañeros, cuyos puntajes presentan un promedio muy cercano a CPS de nivel bajo. Esto podría explicarse que el aumento de conductas insanas puede verse incrementado en los estudiantes por la necesidad que tienen como jóvenes de vivir su libertad fuera de la familia y la influencia de sus compañeros sobre su individualidad.

La religión tampoco influye en las conductas promotoras de salud en este estudio. Sin embargo, los estudiantes que indicaron profesar alguna religión, presentaron un promedio más alto de conducta promotora de salud respecto a los que no indicaron profesar religión. Por el contrario en los estudios: The Relationship Between Spirituality, Health Beliefs, and Health Behaviors in College Students, realizado por Nagel (2007) y Religion and well - Being among canadian university students: the role of faith groups por Frankel, los resultados indicaron que los estudiantes que profesan una fe religiosa, presentaron mejores conductas promotoras de salud que los estudiantes que no tienen alguna filiación religiosa. Los estudiantes que tienen pareja manifiestan mejor conducta promotora de salud que los que no tienen pareja. Sin embargo, este factor tampoco influye significativamente en las conductas promotoras de salud.
Al relacionar la conducta promotora de salud y el nivel de autoestima en los estudiantes de Enfermería, se observa que existe una relación directa y positiva es decir, a mayor autoestima, mayor conducta promotora de salud; resultado que coincide con el estudio realizado en una universidad de Texas por (Chelsea y et al. 2010; citado por Cid, Merino y Stiepovich 2006) realizó un estudio titulado "Can Health Behaviors and Motives Predict College Students' Self-Esteem?", donde se concluye que los motivos de elegir una alimentación saludable y ejercicio físico depende de la autoestima en los universitarios. La autoestima es importante en todos los aspectos de la vida; Branden (1994) considera que es una necesidad básica humana, indispensable para el autodesarrollo normal y sano, y con un valor para la supervivencia, pues se ha relacionado con varios aspectos de salud y del comportamiento.

La relación entre percepción de autoeficacia y conducta promotora de salud, en los estudiantes de Enfermería, resultó altamente significativa, es decir, a medida que aumenta la percepción de autoeficacia, aumenta la conducta promotora de salud de los estudiantes en estudio. La autoeficacia ha sido ampliamente discutida en la literatura de la Psicología Social para explicar la teoría de la motivación y el aprendizaje. Este concepto ha sido adaptado a la Psicología y la Enfermería como un importante predictor de conductas de salud (Kear, 2000). De hecho, frente a lo difícil que puede ser el motivar a la adopción de conductas que promuevan la salud o el de tener conductas nocivas la autoeficacia ha mostrado consistentemente ser un factor de gran importancia. Las investigaciones revelan que altos niveles de autoeficacia tienen consecuencias beneficiosas para el funcionamiento del individuo y su bienestar general (Grembowski, Patrick, Diehr, Durham, Beresford, Kay y Hetcht, 1993; Klein-Hessling, Lohaus y Ball, 2005, citado por Cid, Merino y Stiepovich 2006). Los individuos con alta autoeficacia percibida tienen más probabilidad de evaluar su salud como mejor, estar menos enfermos o depresivos, recuperarse más rápidamente de las enfermedades que las personas con baja autoeficacia (Grembowski et al, 1993). Estos hallazgos tienen importancia e implicaciones para campañas de salud pública y esto sugiere que, el aumentar la autoeficacia, puede ser una estrategia más promisoria en el cumplimiento de las conductas saludables. 


\section{REFERENCIAS BIBLIOGRÁFICAS}

Aspillaga, P. (2003). Resumen Ejecutivo Encuesta Nacional de Salud, 2003. Encuesta Nacional de Salud. Boletín de Vigilancia en Salud Pública de Chile. 8(20), 2-19. Recuperado de: http://epi.minsal.cl/epi/html/elvigia/ VIGIA20.pdf

Cid, P. y Pimenta, A. (2008). Percepción de los beneficios del consumo de drogas y barreras para su abandono entre estudiantes del área de salud. Rev. Latino-am Enfermagem. 16(especial),1-6. Recuperado de: www. revistas.usp.br/rlae/article/download/2528/3047

Cid, P., Merino, J. \& Stiepovich, J. (2006). Factores biológicos y psicosociales predictores del estilo de vida promotor de salud. Revista Médica de Chile. 134(12), 1491-1499. Recuperado de: http://www.scielo.cl/scielo. php?script=sci_arttext\&pid=S0034-98872006001200001

Cid, Patricia \& Orellana,A.(2010). Validación de la escala de autoeficacia general en Chile. Revista Médica de Chile. 138: 551 - 557. Recuperado de: http://www.scielo.cl/ scielo.php?pid=S0034-98872010000500004\&script=sci_ arttext\&tlng $=\mathrm{pt}$

Frankel, G. \& Gail, B. (1994). Religion and well-Being among Canadian University students: The role of faith groups on campus. Journal for the scientific study on Religion. 33(1), 62-65. Recuperado de: http://web.b.ebscohost. $\mathrm{com} /$ ehost/detail?vid=4\&sid=b79f346e-119f-43a 8822e-fe96be5dc43b\%40sessionmgr114\&hid=113\&bdat $\mathrm{a}=$ Jmxhbmc9ZXMmc210ZT1laG9zdC1 saXZl\#db=rfh\& AN=ATLA0000878535

González, R. (2004). Calidad y estilo de vida en estudiantes de enfermería y medicina. Revista del Hospital Psiquiátrico de la Habana. 1(1)1-6. Recuperado de: http:/www.revistahph.sld.cu/hph0104/hph06104.htm

Grembowski, David. (1993). Self-efficacy and behavior among older adults. Journal of Health and Social Behavior. 34(2) 89-104. Recuperado de: http:/www.jstor. org $/$ discover $/ 10.2307 / 2137237$ ?uid=3738800\&uid= $2 \&$ uid $=4 \&$ sid $=21103986339101$

http://www.scielo.cl/scielo.php?pid=S 034 98872006001200001\&script=sci_arttext
Naguel, E. (2007). The Relationship between Spirituality, Health Beliefs, and Health Behaviors in College Students. Journal of religion \& health. 46(1), 141-154. Recuperado de: http://web.b.ebscohost.com/ehost/ detail? $\mathrm{vid}=6 \& \mathrm{sid}=056 \mathrm{a} 6 \mathrm{f} 01-\mathrm{db} 49-4 \mathrm{ffd}-8 \mathrm{a} 04-\mathrm{e} 2 \mathrm{fe} 2 \mathrm{a} 69$ 27 a3\%40sessionmgr114\&hid $=113 \&$ bdata $=$ Jmxhbmc 9 ZXMmc210ZT1laG9zdC1saXZ1

Olivares, S. \& Zacarías, I. (1997). Guía de Alimentación saludable y necesidades nutricionales del adulto. Chile: INTA. Recuperado de: http://www.inta.cl/material educativo/cd/3guiali.pdf

OMS. (2007). Promoción de la Salud: 53 Asamblea Mundial de Salud. Recuperado de: http://apps.who.int/ $\mathrm{gb} /$ archive/pdf_files/WHA53/sa16.pdf

OPS. (2005). Carta de Bangkok para la promoción de la salud en un mundo globalizado. Recuperado de: www. paho.org.spanish/AD/SDE/HS/Carta\%20Bangkok.doc

Pender, N., Walker, S., Sechrist, K. \& Frank, M. (1990). Predicting Health- Promoting. Lifestyles in the Workplace. Nursing Research. 39(6), 326-332. Recuperado de: http://web.b.ebscohost.com/ehost/ detail vid $=4 \&$ sid $=8$ b893250-296b-4e14-a3b9-b368 09560 bad\%40sessionmgr112\&hid $=113 \&$ bdata $=\mathrm{Jm}$ xhbmc9ZXMmc210ZT1laG9zdC1saXZl\#db=mnh\& $\mathrm{AN}=2092305$

Prieto, A. (2003). Modelo de promoción de la salud, con énfasis en actividad física, para una comunidad estudiantil universitaria. Revista de Salud Pública. 5(3) 284-300. Recuperado de: http://www.scielosp.org/scielo.php?script=sci_ arttext\&pid=S0124-00642003000300005 